\title{
Development of a 1.5-tonne niobium gravitational radiational antenna
}

\author{
P. J. Veitch, D. G. Blair, N. P. Linthorne, L. D. Mann, and D. K. Ramm \\ Department of Physics, University of Western Australia, Nedlands 6009, Western Australia
}

(Received 4 March 1987; accepted for publication 22 April 1987)

\begin{abstract}
A 1.5-tonne Nb gravitational radiation antenna is described. Problems associated with a noncontacting magnetically levitated parametric upconverter transducer are discussed, and a system using a bonded microwave reentrant cavity and bonded mechanical impedance transformer is described and analyzed in detail. It is shown that such an antenna can be expected to achieve a noise temperature of $\sim 1 \mathrm{mK}$. An ultralow phase noise tunable microwave source for the transducer pump signal is described, as well as precision bonding techniques which yield a mechanical positioning accuracy of $10^{-6} \mathrm{~m}$, and a reproducibility of $10^{-8} \mathrm{~m}$.
\end{abstract}

\section{INTRODUCTION}

The detection of gravitational radiation will require extremely sensitive antennas with dimensionless strain sensitivities of $\sim 10^{-20}$. This sensitivity is equivalent to a noise temperature of $\sim 10^{-6} \mathrm{~K}$, or a noise number of $\sim 30$, for the present generation of Weber-type resonant bar antennas. (The noise temperature expressed as an equivalent number of quanta at the antenna frequency. A noise number of 1 corresponds to the "standard quantum limit" of linear amplifiers and transducers.) Such a high sensitivity can only be obtained by the use of extremely high acoustic $Q$-factor bars which are cooled to liquid-helium temperatures, and "quantum limited" transducers.

When a burst of gravitational radiation interacts with a resonant bar antenna it induces a small sudden change in the thermal motion of the antenna. This change can only be resolved if it is large compared with the normal fuctuations in the thermai motion of the antenna, wide-band series noise added by the transducer and readout, and narrow-band noise produced by the back action of the transducer on the antenna.

The magnitude of the fuctuations in the thermal motion of the antenna is determined by its $Q$ and physical temperature. The large storage time implied by a high $Q$ restricts the rate at which the thermal motion can vary. By operating the antenna at liquid-helium temperatures the rms amplitude of the thermal motion is decreased. Combining these two properties minimizes the fiuctuations in the thermal motion.

The wide-band noise added by the transducer can be reduced by using long integration times. Integration times longer than the duration of the burst may be used because of the "memory" of the resonant bar antenna. This, however, is at the expense of increased noise due to the thermal motion and back action of the transducer on the bar and reduced bandwidth. The optimum integration time is determined by balancing the contributions from all the noise sources. Ultimately, short integration times will be required to enable directional information to be obtained using antennas located on different parts of the Earth.

The antenna being developed at the University of Western Australia (UWA) uses niobium as the antenna materi- a1, rather than aluminum as used by other groups. The $Q$ factor of the $\mathrm{Nb}$ antenna is sufficiently high that the intrinsic thermal noise of the antenna is negligible at $4 \mathrm{~K}$ and, unlike the Al antennas, it should not need to be cooled to millikelvin temperatures.

Another distinctive feature of the UWA antenna is the transducer. We are developing a parametric upconverter transducer based on a microwave $X$-band reentrant cavity. Parametric transducers have several advantages over the passive transducers being developed by other groups. First, they do not significantly degrade the acoustic $Q$. Also, the operating frequency of the parametric transducer can be chosen to best take advantage of low noise amplifier development. Our choice of the microwave $X$ band enables the transducer to be small yet manageable, and to use ultralow noise cryogenic GaAs MESFET amplifiers and masers as transducer postamplifiers.

The reentrant cavity was initially mounted in a transducer which was magnetically levitated and servocontrolled to its operating position. The noncontacting transducer was designed, primarily, to minimize the acoustic $Q$ degradation of the antenna. Unfortunately, residual low-frequency motion of the transducer caused the power incident on the GaAs MESFET amplifier to vary sufficiently that the noise temperature of the amplifier was degraded. Full details are given later.

We have abandoned the levitated transducer in favor of bonding the reentrant cavity to the antenna. This decision was made after careful investigation of (a) the dependence of the amplifier noise temperature on input power and physical temperature, and (b) the $Q$ degradation caused by bonding small $\mathrm{Nb}$ masses to $\mathrm{Nb}$ resonators.

The present antenna configuration is described in Sec. I of this paper, while the shortcomings of the levitated transducer are discussed in Sec. II. Section III is devoted to an investigation of the projected performance of the antenna in the forthcoming cool down and possible improvements to the antenna. This section contains observations which are relevant to sensitive parametric transducers in general. In the final two sections we discuss the development of an ultralow phase noise tunable microwave source and the bonding of the reentrant cavity to the antenna. 


\section{PRESENT CONFIGURATION}

The resonant bar gravitational radiation antenna being developed at the University of Western Austraiia is shown schematically in Fig. 1. It consists of a 1.5-tonne right-cylindrical $\mathrm{Nb}$ bar to which is bonded a resonant $\mathrm{Nb}$ bending flap tuned to the bar's fundamental longitudinal mode. The bending fip acts as a mechanical transformer, to reduce the output impedance of the bar to the mechanical input impedance of the transducer, which is bonded to the antenna as shown in the figure. The transducer is a microwave $X$-band reentrant cavity.

The antenna is supported by a low-loss, high-isolation two-stage mechanical filter ${ }^{1,2}$ on which the bare bar has an acoustic $Q$ factor of $2.3 \pm 0.3 \times 10^{8}$ in its fundamental longitudiral mode at $5 \mathrm{~K}$. Experiments in low acoustic loss bonding $^{3}$ lead us to expect that the composite antenna, consisting of bar, bending flap, and transducer, should also have a comparable $Q$. At this $Q$ factor the antenna thermal noise is comparable to the "standard quantum limit" for sampling times of a few milliseconds. As discussed below, the antenna thermal noise, is therefore, negligible compared with the present transducer noise, and will not limit the antenna sensitivity until the transducer noise is improved by several orders of magnitude.

The first stage of the support (closest to the antenna) consists of a compact spiral cantilever spring which was cut from a singie piece of commercially pure titanium. This is bolted to a 50-kg mild steel "intermediate mass" which is, in turn, supported by four aluminum cantilevers. Commercially pure Ti was used for the first stage because (a) it has low cryogenic acoustic losses ${ }_{5}^{4}$ and (b) it has almost the same integrated thermal contraction between 300 and $4 \mathrm{~K}$ as $\mathrm{Nb}$, thus preventing differential rotation of the bar relative to the suspension.
The transducer is pumped by an ultralow phase noise signal transmitted to the cavity by a thin (0.020-in.-diam) semi-rigid coaxial cable (UT20). The coaxial cable is bonded to a low-loss three-stage mechanical filter to isolate the antenna from its surroundings. The mechanical isolation is simplified by operating the cavity in reffection, since only one cable must then be used. Operating the cavity in refiection also provides carrier suppression of the microwave power reflected from the cavity (relative to the signal). The latter point will be discussed further below.

Since the transducer is attached to the bar, and is therefore not easily tunable, it is necessary to have a tunable ultralow phase noise source. For this purpose we use a tunable sapphire-loaded superconducting cavity (T-SLOSC), which is tuned to the frequency of the reentrant cavity. A low noise Gunn oscillator is then servocontrolled to the high $Q$ mode of the T-SLOSC, by means of a Pound stabilizer servosystem. ${ }^{5,6}$ This actively stabilized microwave signal is then further stabilized by using the T-SLOSC as a transmission filter. The T-SLOSC is at present contained in another cryostat to facilitate development. It will ultimately be included in the antenna cryostat.

The microwave power reflected from the cavity is phase modulated by the relative motion of the bending flap and reentrant cavity. The carrier reflected from the cavity is, as mentioned above, suppressed relative to the signal sidebands. The amount of suppression obtained is dependent on the microwave coupling to the cavity. While the intrinsic sensitivity of the system is very high, the exiremely small vibration amplitudes $\left(<10^{-16} \mathrm{~m}\right)$ resuit in signal sidebands that are extremely small compared with the reflected carrier.

Carrier suppression is, therefore, necessary to enable amplification of the small signal sidebands, without saturation of the amplifier. (The amplifier used here saturates at an input power $\sim 10^{-6} \mathrm{~W}$.) The first stage of carrier suppres-
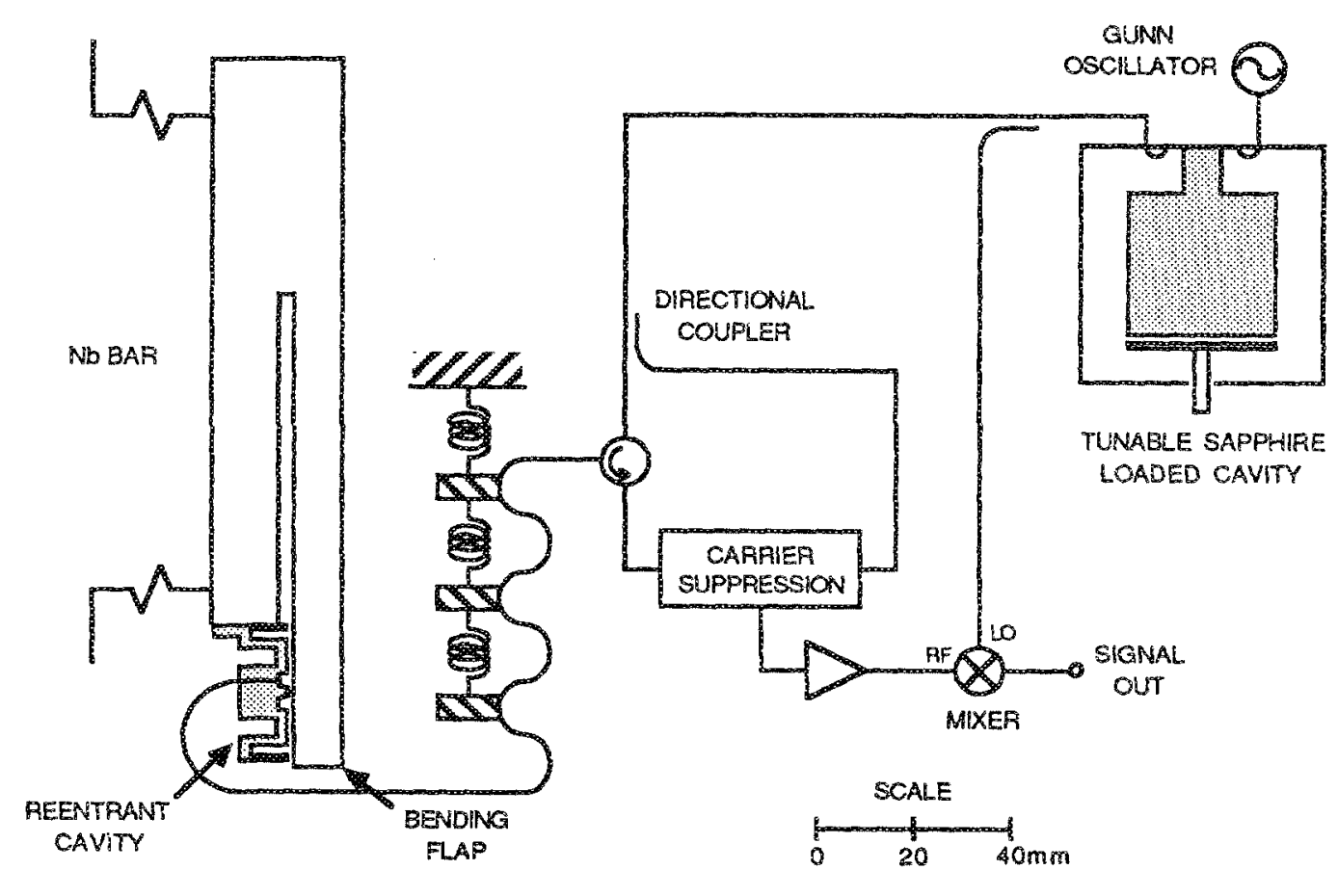

FiG. 1. Schematic of the 1.5-tonne $\mathrm{Nb}$ gravitational radiation an tenna. 


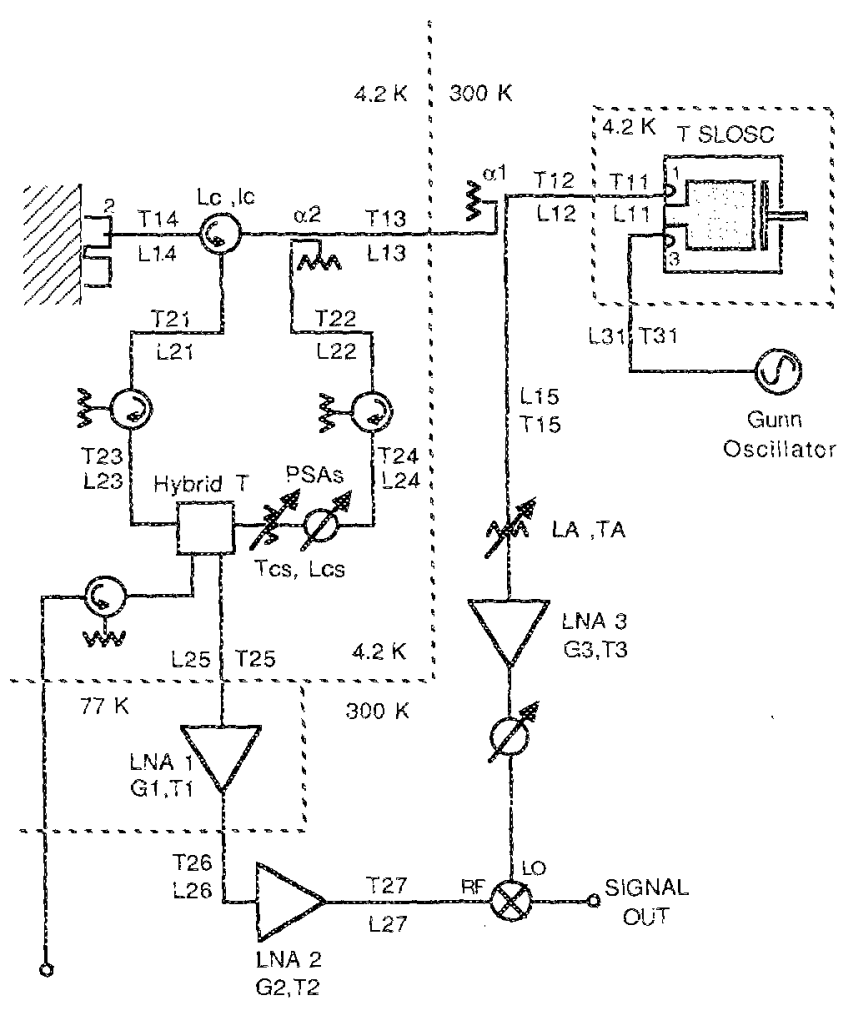

FIG. 2. The microwave circuit used to provide power for the transducer and demodulate the resulting signal. Symbols representing some of the parameters which determine the noise contributed by each element are shown alongsice. The generic symbols $T$ and $L$ represent effective noise temperatures and losses. The symbols $\alpha 1$ and $\alpha 2$ represent directional coupiers.

sion is afforded by setting the microwave coupling close to unity. Because it is impossible to set the coupling with suffdient accuracy, however, a second stage of carrier suppression system must also be used. This additional carrier suppression uses a "hybrid $T$ " (see Fig. 2) to add some unmodulated carrier having almost equal amplitude but opposite phase to the modulated carrier. The phase and ampltude of the unmodulated camier are adjusted using cryogenic phase shifter/attenuators (PSAs) developed in this laboratory. ${ }^{7}$ In this way stable and precise carrier nulling of at least $40 \mathrm{~dB}$ can be achieved.

After carrier suppression the signal is amplificd using a low noise cryogenic GaAs MESFET preamplifer" (LNA1) and a conventional room-temperature low noise amplifier (LNA2). It is then demodulated using a double-balanced mixer (DBM) to recover the signal at the antenna frequency. Unmodulated power from the T-SLOSC is used as the "local oscillator" for the DRM.

The output of the DBM is amplified and then phase sensitively detected at the antenna frequency using a "lock in" amplifier. The two quadratures of the signal are low-pass filtered and sampled every $\tau_{s}$ seconds by a computer; and then processed using a standard digital flter algorithm." Possible events are compared with veto information obtained from seismometers, electromagnetic pulse monitors, and cosmic-ray detectors.

\section{RESULTS USING NONCONTACTING TRANSDUCER}

The antenna configuration described is considerably different from that described previously. ${ }^{12}$ Briefly, in the previous configuration the reentrant cavity was part of a transducer which was magnetically levitated and servocontrolled to its operating position. The operating position was determined by the fixed frequency of a high $Q$ sapphire loaded superconducting cavity resonator (SLOSC). The noncontacting transducer was designed to minimize both the acoustic $Q$ degradation of the antenna, and seismic noise coupling to the antenna. The microwave circuit used was similar to that shown in Fig. 2 except that the SLOSC was included in the antenna cryostat and the GaAs MESFET amplifier was operated at $4.2 \mathrm{~K}$.

In the course of calibration of the antenna sensitivity and through independent measurements on the GaAs amplifier and other identical amplifers at iquid-helium temperatures, we discovered that during the operation of the microwave transducer the effective noise temperature $\Gamma_{A}$ at $\omega_{a}$ $(710 \mathrm{~Hz})$ from the carrier was $\sim 10^{\circ} \mathrm{K}$. This was far in excess of the expected small-signal value $T_{0}$ of $25 \mathrm{~K}$. Upon further investigation the noise was attributed to flicker noise which was enhanced by nonlinearities in the amplifier. It showed a drastic increase as (a) the amplifier physical temperature was decreased below $77 \mathrm{~K}$, and (b) as the input power was increased above $10^{-10} \mathrm{~W}$. Full details of these effects have been reported elsewhere."

The excess noise at "high" microwave power is difficult to avoid in a levitated transducer due to the presence of lowfrequency seismic motions of the transducer. While servocontrol adequately holds the transducer within the $10^{-10}-\mathrm{m}$ band width of the reentrant cavity, residual motion cannot be avoided, due to the presence of $\sim 1 \mu \mathrm{m}$ of seismic motion in the $1-20-\mathrm{Hz}$ range. This motion modulates the amplitude and phase of the carrier reflected from the reentrant cavity, thereby disrupting the carrier suppression and varying the input power to the amplifier. While the input power to the amplifier was still well below the $1-\mathrm{dB}$ gain compression point of the amplifier, it was sufficient to greatly increase the noise temperature. A significant increase in the transducer servocontrol loop gain $(>30 \mathrm{~dB}$ ) would be required to reduce the residual motion to an acceptable level. Due to the presence of high-frequency mechanical resonances it is diffcult to design a transducer for which a servoloop with such high gain would be stable.

Careful analysis of the dependence of $T_{0}$ and $T_{A}$ on physical temperature and power revealed that if the preamplifier could be operated at 77 instead of $4.2 \mathrm{~K}$, and if the input power could be kept below $10^{-9} \mathrm{~W}$ then it would be possible to achieve a $T_{A}$ approaching the small-signal $T_{0}$ value at $77 \mathrm{~K}$ of $40 \mathrm{~K}$. is

\section{NOISE ANALYSIS}

This section starts by discussing some of the parts of Fig. 2 which have so far been omitted. It goes on to make some general observations about the optimization of the microwave "phase bridge" used to demodulate the antenna signal. 
We then examine the antenna sensitivity for several sets of parameters which represent the antenna configuration envisaged for the forthcoming cool down, and several "improved" configurations.

A single microwave transmission line is used to transmit the clean pump signal from the T-SLOSC to the reentrant cavity and to provide power for the carrier suppression. The coupling factor of the directional coupler on this line is small ( $-10 \mathrm{~dB}$ ) since most of the power from the T-SLOSC $\left(\sim 10^{-3} \mathrm{~W}\right)$ is required to operate the room-temperature mixer, whereas only the order of $10^{-5} \mathrm{~W}$ is needed at the reentrant cavity.

Two amplifiers, LNAl and LNA2, are shown in the signal line leading to the rf port of the double-balanced mixer. The latter is necessary to ensure a reasonable level of signal into the mixer, thereby minimizing the mixer voltage noise and local oscillator AM noise, referred to the output of the reentrant cavity. An amplifer (LNA3) is also shown in the local oscillator arm of the circuit, preceded by a variable attenuator. This would be necessary if, for some reason, the tunable SLOSC could not provide sufficient power to drive the mixer.

Alongside each of the microwave circuit elements in Fig. 2 are some of the associated parameters which determine the noise contribution of each element. A number of different possibilities arise in the implementation of such a circuit through the manipulation of these parameters. We have undertaken a study of the noise generated by all of the elements in this circuit and have identified various optimal strategies to be followed for given sets of operational constraints.

In a system as complex as this one, where numerical modeling is essential to calculate changes to the system noise due to altering even a few parameters, it is useful to have an intuitive appreciation of the dominant contributions to the total system noise.

Inspection of Fig. 2 reveals that there should be signifcant cancellation of the phase noise from the T-SLOSC across the double-balanced mixer, and so this should not be a significant source of series noise. The quadrature component of this phase noise, produced by the dispersion of the reentrant cavity does not cancel, on the other hand. The AM noise from the T-SLOSC also contributes noise to the anterna; it produces back action in the reentrant cavity, which is not canceled out in the demodulation. The mixer is also directly sensitive to AM noise because of feedthrough in the mixer and inaccurate adjustment of the phase of the LO signal for quadrature (phase-sensitive) operation of the mixer; this is only a second-order effect, however. Thus, the level of source AM noise, as well as the phase noise, needs to be kept to a minimum. This is accomplished by using a low-noise microwave source and ensuring that the T-SLOSC has a sufficiently high $Q$ that it can filter the transmitted microwave power.

Nyquist noise in the microwave components also contributes uncorrelated phase noise and $\mathrm{AM}$ noise to the system. The components at $4.2 \mathrm{~K}$ contribute insignificantly to this noise compared to those at room temperature and the microwave cables between the antenna and room tempera- ture. Hence, special attention must be given to minimizing line losses $\mathrm{L}_{12}, \mathrm{~L}_{13}, \mathrm{~L}_{15}$, and $\mathrm{L}_{26}$ in Fig. 2. Similarly, if it is necessary to utilize LNA 3 in the LO line, attention must be given to not exacerbating the Nyquist noise through unnecessary saturation of this amplifier.

The components between the output of the hybrid $T$ and the input of the DBM can be combined to define an effective amplifier. The gain and noise temperature of this composite amplifier are critical in determining the overall transducer series noise. It is important to maximize the total gain to minimize the contribution of the mixer voltage noise, which is considerable at the antenna frequency because of ficker noise. In order to achieve the minimum effective noise temperature for this amplifer it is necessary to ensure that LNA 1 does not saturate, necessitating input powers below $10^{-9} \mathrm{~W}$. Some saturation with concomitant enhancement in noise temperature is, however, allowed in LNA2 because of its diminished effect on the effective input noise temperature. One of the complications in determining the effective gain necessary to overcome mixer noise is that the mixer noise is variable (when referred to its input). It depends on the mixer conversion loss, which is itself determined by the level of applied LO drive.

Even if a composite amplifier can be constructed having sufficient gain to negate the noise in the room-temperature mixer it is obvious that a room-temperature mixer (even a very quiet one) still necessitates the use of noisy microwave cables at $300 \mathrm{~K}$ on the LO side, if not on the rf side as well. Consequently, there is an advantage to be gained by using a cryogenic (but not necessarily any lower noise) mixer to eliminate most of the cable Nyquist noise. Of course, in the present circuit configuration there is relatively less to be gained by doing this as long as the T-SLOSC is in another cryostat and is connected by room-temperature lines to the antenna cryostat. Ideally, we would like to have an in situ cryogenic mixer (probably at $77 \mathrm{~K}$ ) and the T-SLOSC mounted near the antenna.

The final critical element in the microwave circuit to which we must give consideration is the reentrant cavity itself. The electrical $Q$ of the cavity determines the size of the upconverted antenna sidebands and so should be as high as possible. At the same time, close to critical microwave coupling is important to provide intrinsic carrier suppression of the reflected, phase-modulated carrier.

We will now consider several diferent scenarios for the present anterna configuration. Some of these scenarios are indicative of the anticipated performance of the antenna during the forthcoming cool down; others are applicable over a longer term, pointing the way for future developments of and improvements to the system. For each scenario we will calculate the total antenna noise, consisting of narrowband antenna thermal and back-action noise, and wide-band series noise. Both the integration time and the level of microwave input power to the T-SLOSC will be regarded as independent variables whose values can be simultaneously determined for the minimization of the total antenna noise. By varying the input power we essentially change the electromechanical coupling between the transducer and the antenna. The optimum power is determined by a balance between the 
TABIE I. Noise analysis sccnarios.

\begin{tabular}{|c|c|c|c|c|}
\hline Parameter/Scenario & 1 & 2 & 3 & 4 \\
\hline Antenna acoustic $Q$ & $5 \times 10^{7}$ & $2 \times 10^{8}$ & $1 \times 10^{7}$ & $2 \times 10^{8}$ \\
\hline T-SLOSC electrical $Q$ & $5 \times 10^{8}$ & $5 \times 10^{7}$ & $5 \times 10^{8}$ & $1 \times 10^{9}$ \\
\hline \multicolumn{5}{|l|}{$\begin{array}{c}\text { Degradation of } \\
\text { T-SLOSC }\end{array}$} \\
\hline phase noise & $\mathrm{OdB}$ & $54 \mathrm{~dB}$ & $0 \mathrm{~dB}$ & $0 \mathrm{~dB}$ \\
\hline amplitude noise & $0 \mathrm{~dB}$ & $14 \mathrm{~dB}$ & $0 \mathrm{~dB}$ & $0 \mathrm{~dB}$ \\
\hline Mixer voitage noise & & & & \\
\hline at $\omega_{a}\left(\mathrm{~V}^{2} / \mathrm{Hz}\right)$ & $8 \times 10^{-17}$ & $8 \times 10^{-17}$ & $8 \times 10^{-17}$ & $4 \times 10^{-18}$ \\
\hline LNA 1 noise & & & & \\
\hline temperatura & $100 \mathrm{~K}$ & $100 \mathrm{~K}$ & $100 \mathrm{~K}$ & $4 K$ \\
\hline ENA1 Gain & 500 & 500 & 500 & 1000 \\
\hline$T_{2 S}$ & $20 \mathrm{~K}$ & $20 K$ & $20 \mathrm{~K}$ & $4.2 \mathrm{~K}$ \\
\hline$L_{-25}$ & $2 \mathrm{~dB}$ & $2 \mathrm{~dB}$ & $2 \mathrm{~dB}$ & $1.5 \mathrm{~dB}$ \\
\hline Optimum sampling & & & & \\
\hline time & $0.14 \mathrm{~s}$ & $7.9 \mathrm{~s}$ & $85 \mathrm{~ms}$ & $86 \mathrm{~ms}$ \\
\hline $\begin{array}{l}\text { Optimum input } \\
\text { power }\end{array}$ & $15 \mathrm{~mW}$ & $110 \mu \mathrm{W}$ & $23 \mathrm{~mW}$ & $5.6 \mathrm{~mW}$ \\
\hline $\begin{array}{l}\text { Electromechanical } \\
\text { coupling }\end{array}$ & $3.3 \times 10^{-3}$ & $2.5 \times 10^{-5}$ & $5.1 \times 10^{3}$ & $5.6 \times 10^{-3}$ \\
\hline $\begin{array}{l}\text { Total noise } \\
\text { number }\end{array}$ & $3.6 \times 10^{4}$ & $1.6 \times 10^{5}$ & $4.2 \times 10^{4}$ & $7.8 \times 10^{3}$ \\
\hline $\begin{array}{l}\text { Total noise } \\
\text { temperature }\end{array}$ & $1.1 \mathrm{mK}$ & $4.9 \mathrm{mK}$ & $1.25 \mathrm{mK}$ & $230 \mu \mathrm{K}$ \\
\hline $\begin{array}{l}\text { Total strain } \\
\text { sensitivity }\end{array}$ & $2 \times 10^{-18}$ & $4 \times 10^{-18}$ & $2 \times 10^{-18}$ & $9 \times 10^{-19}$ \\
\hline
\end{tabular}

series noise, which generally decreases as the power increases, and the back-action noise, which decreases with power. The integration time is adjusted to balance the wideand narrow-band noise terms. The optimization of these two free variables takes account of the energy transfer between the two coupled masses of the bar and bending flap, assuming the detector output is being monitored at only one frequency, halfway between the two coupled mode frequencies.

The simuitaneous optimization with respect to both sampling time and microwave input power leads to a very well-conditioned algorithm. That is, the resultant noise numbers are (at the level of accuracy of interest to us) insensitive to small changes in any of the parameters. Consequently, the confidence in the predictive ability of the algorithm is enhanced if inevitable small deviations of somc experimental parameters from their predetermined or presupposed values are of little consequence to the outcome.

The critical parameters and calculated noise for four scenarios are listed in Table $I$. The reentrant cavity $\mathcal{Q}$ has been kept constant at $2.5 \times 10^{5}$, although this value should probably be able to be increased to about $1 \times 10^{6}$.

Scenario 1 represents a realistic prediction of the antenna performance for the next cool down. The eflective antenna noise temperature of $1.1 \mathrm{mK}$ is achieved with a relatively short sampling time of $0.14 \mathrm{~s}$ and an electromechanical coupling of $3.3 \times 10^{-3}$. Many of the critical parameters have either already been measured or are predicted on the basis of complementary measurements. We are confident that the LNA1 noise temperature value of $100 \mathrm{~K}$ can be achieved as the predicted amplifier input powers are in the vicinity of $2 \times 10^{-10} \mathrm{~W}$. Indeed, the noise temperature may even be less, down to its minimum $T_{0}$ value of $40 \mathrm{~K}$, thus increasing the total antenna bandwidth slightly and reducing the total system noise by approximately $50 \%$.

In scenario 2 we have considered the possibility that the T-SLOSC currently under development severely degrades the purity of the microwave signal through reduced $Q$ and mechanical vibration. The factor of 10 degradation in the $Q$ and the $40 \mathrm{~dB}$ degradation of the phase noise are quite severe, and on the basis of recent measurements are probably overly pessimistic. Despite this, the total system noise temperature has only risen to $4.9 \mathrm{mK}$; but it has done this at the expense of greatly reduced electromechanical coupling $\left(2.5 \times 10^{-5}\right)$ and increased sampling time $(7.9 \mathrm{~s})$.

Scenarios 1 and 2 were evaluated with a high antenna mechanical $Q$ factor: $5 \times 10^{7}$ for the former and $2 \times 10^{8}$ for the latter. (The actual value for scenario 2 does not matter as long as it is $>10^{7}$, because the noise is dominated by additive series and back reaction noise.)

In scenario 3 we consider the case where the antenna $Q$ factor is degraded to $1 \times 10^{7}$. The resulting noise temperature of $1.3 \mathrm{mK}$ is only $20 \%$ higher than that of scenario 1.

Finally, we have considered in scenario 4 a future (and possibly attainable) option in which LNA1 is a near quantum-linited 4-K maser, the T-SLOSC performs as designed, and the antenna mechanical $Q$ approaches $2 \times 10^{8}$. This predicts a total system noise temperature of $230 \mu \mathrm{K}$ with an efiective bandwidth of nearly twice that of scenario 1 . At this level, where improvements reduce the series noise and decrease the optimum sampling times to values close to the energy transfer time for the two coupled modes, the optimization routine becomes dependent on the algorithm used to time average the energy stored in these two modes. This is the limitation imposed by single frequency sampling of the antenna output, as discussed previousiy.

We are currently developing a modified computer algorithm to consider simultancous sampling of the signals at the two coupled antenna mode frequencies, along the lines discussed by Amaldi et al. ${ }^{12}$

\section{TUNABLE SAPPHIRE-LOADED SUPERCONDUCTING CAVITY}

The design of the tunable sapphire-loaded superconducting cavity follows that of the sapphire-loaded superconducting cavities developed in our laboratory. ${ }^{13}$ The SLOSCs have exhibited electrical $Q$ factors as large as $4.2 \times 10^{9}$ at 1.8 $\mathrm{K}$ for $X$-band modes. ${ }^{14}$ These high electrical $Q$ factors can be achieved with relatively modest cleaning and surface preparation of the sapphire resonator and niobium cavity.

The T-SLOSC will, as discussed above, be used as the frequency reference in an ultralow phase noise Pound stabilizer servosystem. Extremely low phase noise in the microwave signal from the $T$-SLOSC is required at carrier offset frequencies near the antenna mechanical resonant frequency $(710 \mathrm{~Hz})$. The T-SLOSC must, therefore, not have any mechanical resonances that could modulate the microwave signal at or near the antenna frequency. It must also be isolated from excess environmental vibrations, and have high mechanical rigidity.

The T-SLOSC is shown schematically in Fig. 3 . It consists of a $30 \times 30-\mathrm{mm}$ cylinder of commercially pure sapphire 


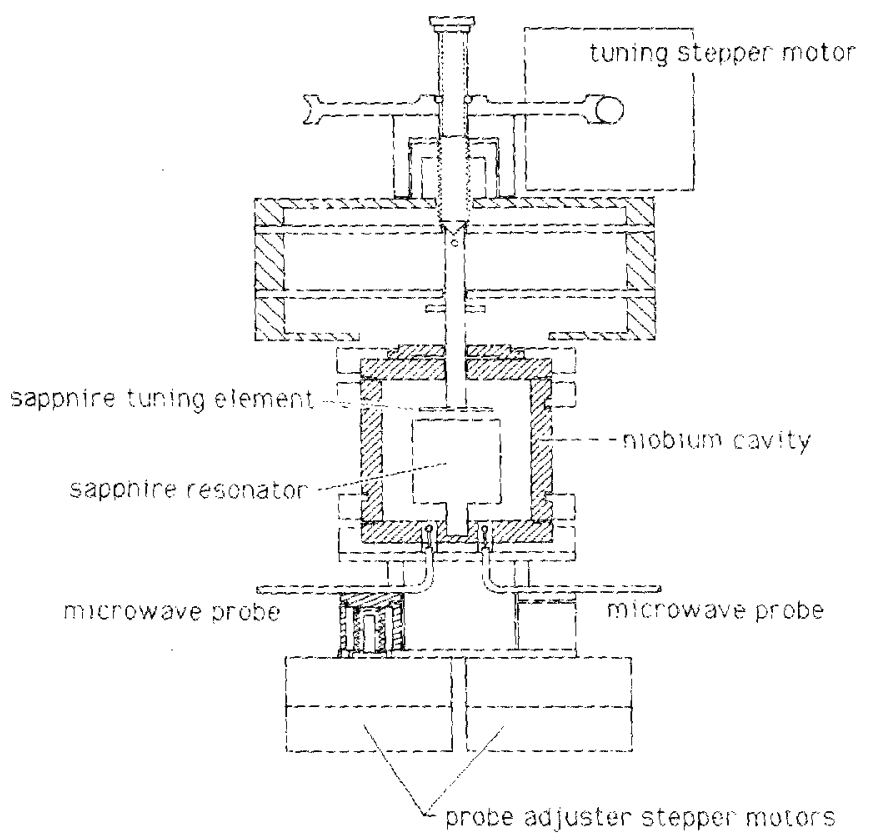

FIG. 3. Schematic of the T-SLOSC.

mounted in a $50 \times 50-\mathrm{mm}$ cylindrical niobium cavity. The sapphire cylinder is supported by a post protruding from its bottom surface which is a tapered fit into a locating hole in the botton of the cavity. The geonetric axis of the sapphire is aligned to within $1^{\circ}$ of the optical axis. The sapphire has a fine ground surface finish.

The T-SLOSC is tuned by adjusting the spacing between a tuning element and the top of the sapphire cylinder. The tuning element consists of a 1.3-mm-thick sapphire disk which has been riveted to a niobium positioning rod. Outside the cavity, the rod locates in a pair of thin metal cantilever diaphragms which are clamped rigidly at their periphery. These diaphragms allow the position of the tuning element to be adjusted while providing rigid transverse support. The position of the sapphire disk is controlled by a leadscrew attached to the niobium rod. The leadscrew is driven by a high-resolution stepper motor through a reduction worm gear. The sapphire tuning element has about a 5-mm dynamic range with about $70 . \mathrm{nm}$ resolution per step.

The microwave signal is coupled into and out of the $T$ SLOSC by two niobium loop probes at the bottom of the cavity. The vertical position of the probes can be independently adjusted by stepper motors mounted below the $T$. SLOSC, allowing the microwave coupling of the probes to be adjusted at cryogenic temperatures. This adjustment is necessary when changing between different modes of the $T$. SLOSC. To prevent $Q$ degradation a superconducting microwave choke is placed around the niobium rod entry hole. Chokes are also used to prevent cross talk between the input and outpat probes.

The prototype T-SLOSC has a high density of modes, with typically about three modes per $100 \mathrm{MHz}$, although some modes appear in more tightly packed groups. Between 9.5 and $10.0 \mathrm{GHz}$ there are 13 modes which have a $Q$ greater than $5 \times 10^{4}$ at $77 \mathrm{~K}$. They provide a frequency coverage of

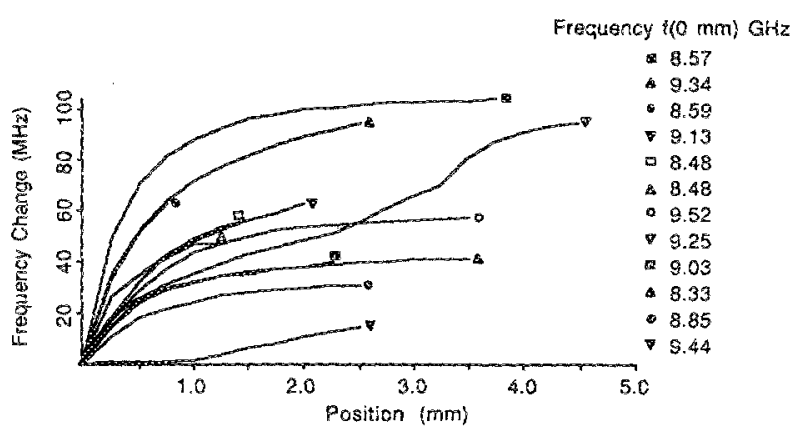

FIG. 4. Typical room temperature T-SLOSC tuning curves. The frequencies $f(0 \mathrm{~mm})$ are those observed when the tuning element is in contact with the resonator. The resonant frequencies generally increase as the tuning element is moved away from the resonator.

$72 \%$ with individual tuning ranges varying from 1 to 78 $\mathrm{MHz}$. A frequency coverage of $43 \%$ is provided by modes having a $Q$ greater than $1 \times 10^{5}$. Most of these modes should satisfy the microwave transducer system noise requirement for a $T$-SLOSC with $Q>5 \times 10^{\top}$ at $4 \mathrm{~K}$. Should the reentrant cavity frequency fall outside the frequency tuning range covered by modes with sufficiently high $Q$ s, it may be necessary to retune the $T$-SLOSC by modifying the sapphire resonator.

Nearly all modes show an exponential type decrease in the tuning coefficient as the tuning element moves away from the sapphire resonator (see Fig. 4). The few modes that do not follow this behavior have lower $Q$ s and are probably "cavity" modes in which a large fraction of the energy is stored in the space between the sapphire and the cavity.

The T-SLOSC has achieved relatively high electrical $Q$ factors at liquid-helium temperatures, with $Q$ s as high as $1.0 \times 10^{9}$ for a mode at $9.7 \mathrm{GHz}$ with a small tuning range of $2.6 \mathrm{MHz}$. Larger, more practical tuning ranges are possible for other modes which have slightly lower $Q$ s.

Ultimately the T-SLOSC will be designed to have high $Q$ mode at the reentrant cavity frequency. The tuning range of this mode must be sufficient to allow for the nonreproducibility of the reentrant cavity frequency in different cool downs $(\sim 5 \mathrm{MH} z$, see Sec. V).

\section{BONDING OF THE REENTRANT CAVITY TO THE ANTENNA}

The accuracy with which the reentrant cavity must be bonded to the antenna is determined primarily by the limited bandwidth of the GaAs MESFET preamplifier; its 9.2-9.9$\mathrm{GHz}$ bandwidth corresponds to a cavity postbending flap separation of approximately $12 \pm 1 \mu \mathrm{m}$. Further, as discussed above, the low-temperature resonant frequency of the cavity should be reproducible, for a given bond, to within the tuning range of the T-SLOSC high $Q$ modes, to enabie optimization of the T.SLOSC.

Preliminary cavity bonding investigations were carried out by gluing a cavity to the end of a "tuning fork" designed to simulate the bending fiap. An equal mixture of liquid epoxy resin and polyamide (" 24 h" epoxy) was used for the bonds, with curing taking place at room temperature. Resonant frequency changes of $400-600 \mathrm{MHz}$ were observed dur- 
ing both the curing of the epoxy and cooling to $77 \mathrm{~K}$. If the glue joint was fiat then a frequency increase of $13 \mathrm{MHz}$, due to the thermal contraction of the niobium, would be expected. The observed frequency changes can be explained by the formation of a wedge-shaped glue joint which subsequently contracts on curing and cooling. The linear contraction of epoxy resin upon curing and cooling to cryogenic temperatures is approximately $0.3 \%$ (Ref. 15) and 0.9\% (Ref. 16), respectively. A misalignment of $0.2^{\circ}$, calculated from the $0.1-\mathrm{mm}$ tolerance in the gluing jig that was used, accounts for the observed frequency shifts.

The gluing jig was abandoned in favor of screwing the cavity to the tuning fork while gluing. Subsequent changes in the resonant frequency of the cavity during curing and cooling were found to be variable in both magnitude and sign. The average magnitudes produced by curing and cooling were reduced to 60 and $200 \mathrm{MHz}$, respectively, which correspond to variations in the cavity postbending flap separation of 0.15 and $0.5 \mu \mathrm{m}$. The observation that the frequency changes during curing and cooling were of the same sign confirmed that wedge-shaped glue joints were still being formed. In the $48 \mathrm{~h}$ that the glue required to cure, the resonant frequency approached its cured value asymptotically, with the major part of the change occurring in the first $6 \mathrm{~h}$. Cooling of the tuning fork did not produce the monotonic change in frequency that would be expected from the contraction of the epoxy resin. Instead, the resonant frequency generally experienced a turning point at abour $100-150 \mathrm{~K}$. This phenomenon cannot be explained by nonuniform thermal contraction resulting from the calculated temperature gradients in either the tuning fork or the glue joint. The frequency change upon warming to room temperature was monotonic. Cryogenic cycling established that the resonant frequency shifts were reproducible to within $5 \mathrm{MHz}$. This corresponds to a change in the cavity-fap separation of 0.01 $\mu \mathrm{m}$.

The above gluing procedure was used for the bending flap. Similar changes in the resonant frequency were observed during curing of the glue and cooling to liquid-nitro- gen temperatures. Further cooling to liquid-helium temperatures resulted in frequency shifts on the same sign and about $5 \%-20 \%$ of the magnitude of the liquid-nitrogen changes. This is consistent with the known thermal contraction of epoxy resin between 77 and $4.2 \mathrm{~K}$.

The final bonding of the cavity to the bending flap yielded a resonant frequency of $9.76 \mathrm{GHz}$ and an unloaded electrical $Q$ of $3.9 \pm 0.4 \times 10^{5}$ at $4.2 \mathrm{~K}$. This $Q$ could be limited by radiation losses since the cavity choke is probably not tuned for this frequency. ${ }^{1}$

\section{ACKNOWLEDGMENTS}

The work was done as part of the Gravitational Radiation Detection Project at the University of Western Australia. The research was supported by the Australian Research Grants Scheme and the Australian Telecommunications and Electronics Research Board.

${ }^{1}$ P. J. Veitch, Ph.D. thesis, University of Western Australia, 1987.

${ }^{2}$ P. J. Veitch, D. G. Blair, M. J. Buckingham, C. Edwards, and F. J. van Kann, IEEE Trans. Mag. MAG-21, 415 (1985).

${ }^{3}$ D. G. Blair and D. K. Ramm, Cryogenics 26, 322 (1986).

${ }^{4}$ P. J. Veitch, J. Ferreirinho, D. G. Blair, and N. Linthorne, Cryogenics (in press).

5S. R. Stein, Ph.D. thesis, Stanford University, HEPL Report 741, 1974

'A. G. Mann and D. G. Blair, J. Phys. D 16, 105 (1983).

${ }^{7}$ L. D. Mann and D. G. Blair, J. Phys. E 16, 119 (1983).

${ }^{8} \mathrm{G}$. Tomassetti, S. Weinreb, and $\mathrm{K}$. Wellington, Electron. Lett. 17, 949 (198i).

${ }^{9}$ P. Bonifazi, V. Ferrari, S. Frasca, G. V. Pallottino, and G. Pizzella, 1 Nuovo Cimento 1C, 465 (1978).

${ }^{10}$ L. D. Mann, D. G. Blair, and K. J. Wellington, Electron, Lett. 22, 1037 (1986).

${ }^{11}$ L. D. Mann, Ph.D. thesis, University of Western Australia (submitted).

${ }^{12}$ E. Amaldi, G. Pizzella, P. Rapagnani, F. Ricci, P. Bonifazi, G. Cavallari, E. Coccia, and G. V. Pallottino, Il Nuovo Cimento 9C, 51 (1986).

${ }^{13}$ D. G. Blair and S. K. Jones, IEEE Trans. Mag. MAG-21, 142 (1985).

${ }^{4}$ S. K. Jones and D. G. Blair, Electron. Lett. (submitted).

${ }^{15}$ R. L. Patrick, Treatise on Adhesion and Adhesives (Dekker, New York, 1969), Vol. 2.

i6Y. S. Touloukian and E. H. Buyco, Thermophysical Properties of Matter (Plenum, New York, 1970), Vol. 13. 